\title{
ORIGINAL ARTICLE \\ Dissociations in the meaning of risk between health-care professionals and individuals with spinal cord injury
}

\author{
M Eijkholt ${ }^{1}$, BK Kwon ${ }^{2}$ and J Illes ${ }^{1}$
}

Objective: Risks have been a central concern in stem cell research overall, and in clinical trials of individuals with spinal cord injury (ISCIs) in particular. We sought to elucidate how two important stakeholder groups-health-care professionals (HCPs) and ISCIs_-view and value both the physical and non-physical risks of stem cell interventions.

Setting: The study was conducted in Canada, and included participants from both Canada and the United States America.

Study design: We used semi-structured interviews to gain perspectives on risk from HCPS and ISCls.

Methods: We applied a constant comparative analytic strategy to derive themes from the discourse collected through the interviews. Results: We identified three major themes about risk from $12 \mathrm{HCP}$ and $24 \mathrm{ISCl}$ participants: focus, rationale and approach. The salient components of the themes differed: HCPs focus on the physical causes of risks, and the ISCls on their downstream consequences as well as on non-physical risks; HCPs are concerned about evidence, and ISCls about experience; and HCPs approach risk narrowly, whereas the approach of ISCIs is more broad and contextualized.

Conclusion: Although major themes were common to the two stakeholder groups, the components of the themes were dissociable and illustrate differences in what HCPs and ISCls worry about, why they worry and how they approach their worries. We draw upon these findings to make recommendations for improving risk communication and informed consent for stem cell research for spinal cord injury.

Spinal Cord (2013) 51, 909-912; doi:10.1038/sc.2013.103; published online 17 September 2013

Keywords: spinal cord injury; stem cells; risk; ethics; communication; consent

\section{INTRODUCTION}

Clinical trials for spinal cord injuries (SCIs) are being initiated with increasing frequency and, as stem cell technologies steadily develop and evolve, it is necessary to carefully consider the risks of such trials. The advancement of research requires an understanding of the multidimensionality of risk as viewed by all stakeholders. The importance of risk studies designed to achieve this goal has been eloquently described for gene-based therapies and may be seamlessly translated to stem cell research: 'If the field is to make progress, it is necessary to understand how risk is defined... how understandings of risk differ, how risk is assessed, how decisions about risk are made, and how ... risks are communicated to subjects and research participants during the informed consent process.'

Risks have been a central concern in stem cell research overall, and in clinical trials of individuals with SCI (ISCIs) in particular. Risks of novel interventions in these types of trials are not 'strictly a private matter. ${ }^{2}$ Rather, they have an impact on scientific discovery broadly, given the still largely uncharted territory for human translation in research, clinical and regulatory communities alike. Regarding the risks of stem cell therapies for SCI, the scientific literature and regulatory oversight have focused primarily on medical and physical considerations, such as the risk of infection, cancer or neurologic worsening. ${ }^{3,4}$ However, risk is a complex concept with different meanings, definitions and layers. ${ }^{5,6}$ Academic papers dissect risks according to different definitions, phrasing risks in terms of an unwanted event, the cause of an unwanted event or a probability formula. ${ }^{7,8}$ They analyze risks within levels of uncertainty, ${ }^{9,10}$ and consider, on a theoretical level, and without reference to a practical definition, which risks would be acceptable to the SCI population on micro, meso and macro levels. ${ }^{9}, 11,12$ The micro level is defined by relationships between researchers, physicians and their participants, the meso level by oversight from institutional review boards and ethics committees, and the macro level by regulatory structures such as those from the Food and Drug Administration and other government bodies.

The conceptually focused lens ignores important practical components that are critical for informed decision making in practice, such as issues how risks are approached and weighed by the different stakeholders. For example, if there is a risk of neurologic deterioration after stem cell transplantation, do the ISCIs who incur this risk worry about it to the same degree and for the same reasons as the researchers studying the technology? There is evidence that risk perceptions vary between professionals and patients, and may be influenced by both hope ${ }^{12}$ and hype in the stem cell context. ${ }^{13-17}$ Studies of risk communication generally speak to the need for incorporating and addressing personal values in the informed

${ }^{1}$ Canada Research Chair in Neuroethics, Division of Neurology, Department of Medicine, National Core for Neuroethics, University of British Columbia, Vancouver, British Coulmbia, Canada and ${ }^{2}$ Canada Research Chair in Spinal Cord Injury, Combined Neurosurgical and Orthopaedic Spine Program (CNOSP), Department of Orthopaedics, University of British Columbia, Blusson Spinal Cord Center, Vancouver, British Columbia, Canada

Correspondence: Dr J Illes, Division of Neurology, Department of Medicine, National Core for Neuroethics, University of British Columbia, 2211 Wesbrook Mall, Koerner S124, Vancouver, British Columbia, Canada V6T 2B5.

E-mail: jilles@mail.ubc.ca

Received 17 May 2013; revised 25 July 2013; accepted 28 July 2013; published online 17 September 2013 
consent process. ${ }^{13,18}$ However, such studies have not focused specifically on ISCIs. In particular, none have directly compared how risk perceptions differ between the two stakeholders.

Previous work from our own group has revealed that while risks are an important consideration in an individual's receptivity and willingness to consider participating in stem cell trials, such perspectives shift over time. ${ }^{12,14-16}$ Our results underscored the need to consider a patient's perception of risk as a dynamic rather than static phenomenon when designing clinical trials. Further research has interrogated the views of health-care professionals (HCPs) and injured individuals about the evidence needed to move from bench to bedside considering different risk frameworks. ${ }^{16}$ These empirical efforts have established a connection between hopes for recovery and risk tolerance of ISCIs, and substantiate the need for better risk communication to optimize informed consent processes. ${ }^{19}$

The present study continues our efforts to elucidate how HCPs and ISCIs approach the risks of stem cell interventions for SCI. We sought to understand how they view and consider risks, without a fixed prior definition. By understanding the perspectives of these two important stakeholders, it is possible to improve the risk communication process beyond the current state-of-the-art, and to provide both a better top-down and a bottom-up approach in decisions about research participation and research development.

\section{MATERIALS AND METHODS}

\section{Recruitment}

We recruited HCPs and ISCIs from Canada and the United States of America via open advertisements and mailings between March and July 2012. We also placed invitations to participate in the study on the websites of the Canadian Paraplegic Association, the Rick Hansen Institute and the Canadian Spine Society. Announcements were posted at the Interdependence 2012 Conference, the Annual Canadian Stem Cell Network Meeting and at the International Collaboration On Repair Discoveries (ICORD) in Vancouver, Canada.

Eligibility for ISCIs required that they were English speaking, at least 19 years of age and more than 1-month post injury. Individuals were excluded if they had actually undergone a cell transplant for their SCI. Eligibility for HCPs was defined by their research with stem cells or through professional involvement with injured individuals, and also required English fluency. All eligible participants who signaled interest in this study were sent a letter of information and a consent form before enrolment.

\section{Data acquisition}

We conducted semi-structured interviews in person or by telephone to probe participants' views about the risks of stem cell interventions. All interviews were audio recorded, transcribed verbatim and made software-ready (NVivo, version 8, QSR, Doncaster, Victoria, Australia). For both the HCPs and ISCIs, the conduct of the interviews adhered to a guide that was piloted, refined and informed by feedback from collaborators in methodology, experts in stem cell biology and ISCIs. The interviews lasted between 30 and $80 \mathrm{~min}$. The first few questions were general and probed for perceptions on the main risks of stem cell interventions. For example: 'Could you tell me what you know about the risks of stem cell interventions for spinal cord injury? What do you see as the risks of participating in a stem cell trial?' Subsequent questions were more focused on perceptions of physical risks such as cancer and pain. For example: 'Scientists have discussed cancer as a potential risk of stem cell interventions for SCI. What do you think about such concerns?' Further questions addressed non-physical risks such as social or psychological risks. For example: 'Some people have talked about economic risks, such as the financial implications of participating in research or receiving stem cells, jeopardized insurance, or lost benefits. What do you think about such concerns?'

\section{Analysis}

The interviews yielded 45 total hours of audio recordings for analysis and more than 3400 units of data. We used the qualitative methodology of constant comparison to analyze the data, whereby two researchers independently examined the transcriptions. Both coders (co-author ME and a trained research assistant) possessed practical experience in qualitative research methods. ${ }^{20}$ The inductive analytic process involved segmenting, labeling and coding raw data, while searching for patterns of intersection. The process was interpretive and iterative, bringing critical analysis and conceptualization of results into textual themes. ${ }^{21}$ Dominant themes emerged from the triangulation of all aspects of the analysis and conceptual relationships of the data with theories of risk and risk perception. ${ }^{22,23}$ Discrepancies between coders were highlighted and discussed until consensus was achieved. ${ }^{24}$ All themes established in the early phases of data gathering were revisited continuously throughout the course of data collection and analysis and until data saturation was achieved. ${ }^{20}$

The final thematic results combined observations and interpretations, and moved the data from observed phenomena to the generation of theory. Representative quotations from HCPs and ISCIs around specific themes that emerged are provided in the Results section.

\section{Statement of ethics}

The authors' institutional review board approved the study. The authors certify that all applicable institutional and government regulations concerning the ethical use of human volunteers were followed during the course of this research.

\section{RESULTS}

Twelve HCPs and thirty ISCIs responded to our recruitment efforts and expressed interest in participating in this study. Ultimately, we obtained consent to participate and interviews from all 12 HCPs and 24 of the 30 ISCIs. Six ISCIs could not be reached for follow-up after initially signaling interest. The interviews were one-time events and no one dropped out or terminated the interview early.

The 12 HCPs included 7 physicians, 3 of whom additionally conducted basic science research on stem cells and 5 allied HCPs with a nursing or physiotherapy background. There were eight women and four men in this group; mean age of 47 years. The 24 ISCIs included 6 women and 18 men, with a mean age of 46 years. The ISCIs were, on average, 7.4 years post cervical, ranging from 1.5 to 22 years post injury. Fourteen had cervical injuries and ten had thoracic injuries. Eleven were married, six were single, and seven were divorced.

Our analysis generated three major common themes across the HCP and ISCI groups: (1) focus: what risks they worry about, (2) rationale: why they worry and (3) approach: how they deal with their worries. These themes were not exclusive to the particular stakeholder group, but were dominant aspects of the discussion of risk for each (Table 1).

Table 1 Major emergent themes

\begin{tabular}{lll}
\hline & HCPS $(\mathrm{N}=12)$ & ISCls $(\mathrm{N}=24)$ \\
\hline \multirow{2}{*}{ FOCUS } & Cause & Consequence \\
& Delivery & Suffering \\
& Adverse biological effects & Long-term health and social effects \\
RATIONALE & Evidence & Experience \\
& Animal models & Vulnerability and management \\
& Statistics & Impact on family \\
APPROACH & Narrow & Broad \\
& Compartmentalized & Contextualized \\
& Dispassionate & Emotional
\end{tabular}

Abbreviations: $\mathrm{HCP}$, health-care professional; ISCI, individuals with a spinal cord injury. 


\section{Focus: what they worry about}

The two stakeholder groups differed in the focus of what they worried about. HCPs were concerned with causal events such as the direct injection of the cells into the spinal cord and the biological activity of the cells once transplanted such as tumor growth. For example,

' ... The main risk to that is discussed or known is the risk of teratoma. There's an additional risk of the delivery method for spinal cord in particular ...' (HCP \#09).

By contrast, ISCIs worried more about the downstream consequences of such events. They were concerned with consequences that would affect their lives, particularly around the potential for longterm suffering. For example,

'... I think if your immune system is way down and you're in a wheelchair, you're just more likely to have ... endless problems.' (ISCI \#07).

ISCIs also focused more on non-physical risks such as social risks (loss of friends, loss of job or impact on care givers) and economic risks (loss of income, loss of benefits and other financial costs). For example, when considering the economic risks associated with trial participation, one participant offered,

'It would inhibit me from participating at all period. I mean, there's just no way I could afford to pay any more or jeopardize my family's finances any more.' (ISCI \#14).

Although HCPs also signaled appreciation for suffering and other downstream consequences, this was not the dominant focus of their concerns.

Although less prevalent, there were also comments from the ISCIs about psychological risks (loss of hope, anger and disappointment). This contrasted sharply with HCPs who generally did not express concerns with such non-physical risks. For example,

'I don't think they're that great... The patients know that when they sign up...' (HCP \#12).

Although HCPs were reserved on the matter of non-physical risks, they expressed explicit concern about participatory risk: where individuals who choose to enter a stem cell trial would be excluded from other trials, conducted concurrently or in the future, and precluded from associated potential benefits of more advanced and possibly more efficacious stem cell technologies. For example,

'I think it's (participatory risk) highly relevant. I would not include a person in a clinical trial with cell-based therapy if they had previously received a cell-based therapy.' (HCP \#03).

\section{Rationale: why they worry}

The nature of risk was a second dominant theme that differed between the two cohorts. To define the nature of risks, HCPs focused on scientific evidence, such as that obtained from animal models and statistics. For example,

'It's difficult to assess in an animal model because we can't assess (risk) with the same sensitivity that we can in a human.' (HCP \#09).

By contrast, ISCIs focused on the experiential impact, their vulnerability and the manageability of the unwanted event. For example,

'I don't want to get better and be sick all the time, kind of a thing.' (ISI \#07)

ISCIs also spoke about experiential impact in terms of issues such as independence and interaction with family members. For example,

'... if I was single and unattached without children and family then it would be different.' (ISCI \#09).

\section{Approach: how they approach their worries}

Alongside the differences between what HCPs and ISCIs worry about and why they worry about them, we also discerned differences in their approach to risk. HCPs approached risks in a narrow way, compartmentalizing them and reasoning authoritatively but dispassionately. For example,

'... inform them what the problem could potentially be, and then inform them of what you might potentially do to counteract that problem. And that's it. I think once you've informed them, the decision is theirs.' (HCP \#02).

The perspective of the ISCIs was more broad, contextualized and emotional. An important strategy among ISCIs was to approach the risks as a trade-off. For example,

'I would rather risk death and be able to regain some function and be more independent...' (IND \#17).

and

'I don't want my suffering-I don't want my family to suffer more than what they've already suffered with my injuries. It's taken a toll on everybody....' (ISCI \#03).

\section{DISCUSSION}

Risk is a key issue in the development of research involving human subjects overall, and for stem cell research involving ISCIs in particular. ${ }^{9}{ }^{12,14-16}$ Definitions and meanings of risk vary, however, and understanding these in terms of hazard, exposure, consequence and probability is complex. ${ }^{6}$ In this study, we identified three major differences in perspectives about risk between HCPs and ISCIs.

Prior research data from our team have already highlighted that risk perspectives of ISCIs develop over time post injury, ${ }^{12,15}$ and other researchers have demonstrated that hope, in general, influences receptivity of stem cell research. ${ }^{17}$ Differences in risk perspectives between professionals and non-professionals or patients have been identified by others, ${ }^{13,25,26}$ who have underscored the need to understand this phenomena and determine which risks are acceptable to the various stakeholders and why. ${ }^{27-29}$ The data here confirm these findings and provide insights that specifically extend the understanding of risk perceptions of stakeholders for stem cell interventions in SCI.

We identified differences revolving around the focus, rationale and approach to risk. Focus dissociates by cause and consequence; rationale by evidence and experience; and approach by breadth and depth of concerns about risks. HCPs reflect on risks in terms of causal events, whereas ISCIs worry about risks in terms of their downstream consequences. HCPs consider risk in terms of evidence and statistical measurability, and the uncertainty inherent in the procedure or technology. ISCIs describe their concerns about risks based on the nature of their potential overall experience. This is embedded in a context of potential impact and vulnerability that extends beyond the novelty of a procedure and statistical likelihood of its success. Consistent with the first two dominant themes, the perspectives of HCPs are narrow and calculated; that of ISCIs is contextualized within their life circumstances as a whole.

This study engaged HCPs of varying specialities and ISCIs with varying levels, severities and chronicities of injury. Familiarity with clinical or biological aspects of stem cell research was deliberately not specified or standardized. Despite this, the overall results reflect a consistent spectrum of views about risk from HCPs and ISCIs. When probed in depth, our results confirm the complex contents of risk in the stakeholders' views. Even though we reached data saturation in the analysis, we recognize that our cohorts of HCPs and ISCIs were relatively small. Such sample sizes, however, are not uncommon in 
qualitative research studies. We also acknowledge that a limitation inherent to such qualitative research is coder bias, even in the face of systematic analyses and consensus.

This initiative sought to characterize the perspectives of risk between health-care providers and ISCIs on the topic of stem cell interventions. Our analyses depict considerable dissociations between the two stakeholders' approaches or meanings of such risks. The natural question that arises is how awareness of these dissociations might inform the risk communication process in stem cell clinical trials. Effective risk communication should seek to convey a wide understanding about cause and consequences, shortand long-term impact, and both physical and non-physical phenomena. Although we did not directly address the topic of risk communication in our study, a number of considerations emerge from the data. First, our findings underscore that a discussion of physical risks in terms of a purely causal events and isolated variables, whereas obligatory from the researchers' perspective, may not capture what is truly meaningful to potential subjects. Nonphysical risks, in particular, appear to be highly relevant to ISCIs, and are either overlooked or underemphasized by HCPs. Second, our findings reveal that the reasons why certain risks are worrisome differ between the stakeholders. Those engaged in risk communication would be well served to be aware that such differences may exist and to pay particular attention to understanding them. Finally, our findings demonstrate that risk is not a neutral term, but value laden. Meaningful risk communication requires a combination of both science and humanity-especially so in the clinical translation of stem cell research that is under constant public scrutiny, and where informed consent is a delicate matter.

\section{DATA ARCHIVING}

There were no data to deposit.

\section{CONFLICT OF INTEREST}

The authors declare no conflict of interest.

\section{ACKNOWLEDGEMENTS}

This study was supported by SCN/Research: 12/5226 (PP59) (JI). We thank Mr Rick Hansen, Dr Rob Carlson, Dr Marcel Dvorak, Ms Doramy Ehling, Ms Debbe Heayn, Dr Jane Roskams, Dr Wolfram Tetzlaff, Dr David Whitehurst for their expert contributions. We are also grateful to Sophia Park for her help with the analysis and Vera Khramova for her help with the manuscript. Dr Marleen Eijkholt is currently affiliated with the Alden March Bioethics Institute, Albany Medical College, New York.
1 Deakin CT, Alexander IE, Kerridge I. Accepting risk in clinical research: is the gene therapy field becoming too risk-averse? Mol Ther 2009; 17: 1842-1848.

2 Kimmelman J. Tomorrow, interrupted? Risk, ethics, and medical advance in gene transfer. Mol Ther 2009; 17: 1838-1839.

3 Owens J. Stem-cell treatments for spinal-cord injury may be worth the risk. Nature 2009; 458: 1101

4 Herberts C, Kwa MS, Hermsen HP. Risk factors in the development of stem cell therapy. J Transl Med 2011; 9: 29.

5 Warings $D$, Lemmens $T$. Integrating values in risk analysis of biomedical research: the case for regulatory and law reform. Univ Tor Law J 2004; 54: 249-290.

6 Rid A, Wendler DA. Framework for risk-benefit evaluations in biomedical research. Kennedy Inst Ethics J 2011; 21: 141-179.

7 Ropeik D, Gray G. Risk. A Practical Guide for Deciding What's Really Safe and What's Really Dangerous in the World Around You. Houghton Miffin Company: Boston, 2002.

8 Weijer C. The ethical analysis of risk. J Law Med Ethics 2000; 28: 344-361.

9 Solbakk JH, Zoloth L. The tragedy of translation: the case of 'First Use' in human embryonic stem cell research. Cell Stem Cell 2011; 8: 479-481.

10 Sahlin N, Persson J, Vareman N. Unruhe und Ungewissheit: Stem Cells and Risks In Hug K and Hermerén G (eds). Humana Press: Totowa, NJ, 2011, p 421-429.

11 Bretzner F, Gilbert F, Baylis F. Brownstone RM Target populations for first-in-human embryonic stem cell research in spinal cord injury. Cell Stem Cell 2011; 8: 468-475.

12 Illes J, Reimer JC, Kwon BK. Stem cell clinical trials for spinal cord injury: readiness, reluctance, redefinition. Stem Cell Rev 2011; 7: 997-1005.

13 Slevin ML, Stubbs L, Plant HJ, Wilson P, Gregory WM, Armes PJ et al. Attitudes to chemotherapy: comparing views of patients with cancer with those of doctors, nurses, and general public. BMJ 1990; 300: 1458.

14 Eijkholt M, Kwon BK, Illes J. Decision-making in stem cell trials for spinal cord injury: the role of networks and peers. Regen Med 2012; 7: 513-522.

15 Kwon BK, Ghag A, Dvorak MF, Tetzlaff W, Illes J. Expectations of benefit and tolerance to risk of individuals with spinal cord injury regarding potential participation in clinical trials. J Neurotrauma 2012; 29: 2727-2737.

16 Kwon BK, Ghag A, Reichl L, Dvorak MF, Illes J, Tetzlaff W. Opinions on the preclinical evaluation of novel therapies for spinal cord injury: a comparison between researchers and spinal cord-injured individuals. J Neurotrauma 2012; 29: 2367-2374.

17 Scott C, DeRouen M, Crawley L. The language of hope: therapeutic intent in stem-cell clinical trials. AJOB Prim Res 2010; 1: 4-11.

18 O'Connor AM, Légaré F, Stacey D. Risk Communication in Practice: The Contribution of Decision Aids. Br Med J 2003; 327: 736-740.

19 Longstaff H, McDonald M, Bailey J. Communicating risks and benefits about ethically controversial topics: the case of induced pluripotent stem (iPS) cells. Stem Cell Rev 2012: 1-9.

20 Glaser B, Strauss A. The Discovery of Grounded Theory: Strategies for Qualitative Research. Aldine Publishing: New YorkCompany, 1967.

21 Glaser B. The constant comparative method of qualitative analysis. Soc Prob/ 1965 12: $436-455$.

22 Charmaz K. Constructing Grounded Theory: A Practical Guide Through Qualitative Analysis, Sage Publications Limited, 2006.

23 Ryan GW, Bernard HR. Techniques to identify themes. Field methods 2003; 15 : 85-109.

24 Thorne S, Kirkham SR, Columbia B, Flynn-magee KO. The analytic challenge in interpretive description. Int J Qual Methods 2008: 1-11.

25 Sjöberg L.. Risk perception by the public and by experts: a dilemma in risk management. Hum Ecol Rev 1999; 6: 1-9.

26 Nelkin D. Communicating technological risk: the social construction of risk perception. Ann Rev Public Health 1989; 10: 95-113.

27 Kachuck N. When neurologist and patients disagree on reasonable risk: new challenges in prescribing for patients with multiple sclerosis. Neuropsychiatr Dis Treat 2011; 7: 197-208.

28 Master Z, McLeod M, Mendez I. Benefits, risks and ethical considerations in translation of stem cell research to clinical applications in Parkinson's disease. J Med Ethics 2007; 33: 169-173.

29 Goldring CE, Duffy PA, Benvenisty N, Andrews PW, Ben-David U, Eakins R et al. Assessing the safety of stem cell therapeutics. Cell Stem Cell 2011; 8: 618-628. 